\title{
Towards the merger of Hawking radiating black holes
}

\author{
Huiquan $\mathrm{Li}^{1}$ and Jiancheng Wang \\ Yunnan Observatories, Chinese Academy of Sciences, \\ 650216 Kunming, China \\ Key Laboratory for the Structure and Evolution of Celestial Objects, \\ Chinese Academy of Sciences, 650216 Kunming, China \\ Center for Astronomical Mega-Science, Chinese Academy of Sciences, \\ 100012 Beijing, China
}

\begin{abstract}
We discuss the merger process of binary black holes with Hawking radiation taken into account. Besides the redshifted radiation to infinity, binary black holes can exchange radiation between themselves, which is first redshifted and then blueshifted when it propagates from one hole to the other. The exchange rate should be large when the temperature-divergent horizons are penetrating each other to form a single horizon with unique temperature. This will cause non-negligible mass and angular momentum transfer between the black holes during the merging process of the horizons. We further argue in the large mass ratio limit that the light hole whose local evaporation is enhanced by the competing redshift-blueshift effects will probably evaporate or decay completely before reaching the the horizon of the heavy one. We also discuss the possibility of testing Hawking radiation and even exploring the information loss puzzle in gravitational wave observations.
\end{abstract}

\section{Introduction}

The gravitational science has been brought into a new era since the first observation of gravitational waves (GWs) from binary black holes by the LIGO/Virgo collaborations $[1,2]$. The successful discovery opens a new window for the observational channels. It provids a powerful tool to probe the properties of black holes.

The confirmation of a GW event needs the match of the signal with a large amount of waveform templates. The physical parameters of the original black holes and the final

\footnotetext{
${ }^{1}$ E-mail: lhq@ynao.ac.cn
} 
one are meanwhile inferred from the matched waveform. The templates are evaluated in general relativity with a combination of the analytical and numerical methods. This is usually based on the classical mechanics of the binary system, in which the black holes are usually taken as point-like particles that only have masses and spins.

However, black holes are objects of finite size with an event horizon that can radiate. The black hole mechanics takes close analogy to the thermodynamical laws. This inspires the findings that black holes have the thermal features, like entropy and temperature. In the semiclassical theory, it was shown by Hawking [3] that black holes can radiate in the black body spectrum from their event horizons. So black holes should evaporate and evolve due to the Hawking radiation. As observed far from the hole, the Hawking temperature is quite small and negligible for a massive black hole so that it has a long lifetime $[4,5]$.

It has been pointed out that the mechanical laws for isolated black holes can be extended to the case of multiple black hole systems [6]. This is further verified in various examinations in post-Newtonian (PN) and field theory approaches [7, 8, 9, 10]. It implies that thermal features should still hold in multiple black holes. The black holes should also evolve due to radiation. It is easy to find that, except for the radiation escaping to infinity, the black holes in the multiple system could exchange Hawking radiation among themselves.

So, involving the quantum features of black holes, we should take Hawking radiation into account in considering the dynamics and GW emission of binary black holes, as suggested in [11]. Of course, the Hawking radiation from a massive black hole is quite weak as observed just some distance away from the horizon. The thermal particles emitted from the event horizon will be mostly reflected back by the curvature of the spacetime and only very few of them with high energies can escape from the near-horizon region. But the radiation exchanged between the black holes would be not necessarily weak, in particular when the black holes get very close to each other. For a stationary black hole, the local Hawking temperature increases as distance decreases and diverges at exactly the horizon. So, when considering the merger of black holes, we must pay additional attention on the crossing process of the temperature-divergent horizons, during which the exchange of thermal particles could be non-negligible.

This can also be understood in the "thermal atmosphere" scenario [12]. Due to Hawking radiation, the space around a black hole is not empty for local observers near the horizon in the semiclassical theory. In the membrane paradigm, the thermal particles emitted and trapped in the narrow layer just above the horizon form a "thermal atmosphere", which can be viewed as a surrogate of the black hole. It recodes all the information 
of the hole in its history, including the mass, angular momentum and entropy. So the merging process of binary black holes can be viewed as the mixing process of two thermal subsystems. There should exist mass and angular-momentum transfer between the two atmospheres.

The exchange of Hawking radiation will cause the black hole parameters to vary with time. In contrast to the classical theory case, the masses and angular momenta of the black holes may not be constant any more. In particular, the variation of the black hole parameters should become quite prominent when the temperature-divergent horizons are penetrating each other. The time-varying parameters should affect the waveforms of GWs emitted from binary black holes with different mass ratios. So we might discriminate these features from the GW signals and test the Hawking radiation effects.

However, the aim to tackle exact Hawking radiation from binary black holes is almost impossible at the moment since the accurate spacetime is unknown. In this work, we make some simple analysis by assuming that Hawking radiation is a thermal feature associated with the event horizons, which are though hard to be located in a dynamical system. The radiation flux between black holes is determined with analysis of the redshift factor on the spacetime. In the dynamical spacetime of the binary system, the redshift factor associated with the Killing vectors is usually not derivable. But, we think that there could be two situations that discussion can be simplified. The first is the case when the black holes are separated by a large distance. The other case is the one of a binary system with large mass ratio. In such a system, the various corrections to the redshift factor can be dealt with in a controllable way, though we only consider the probe approximation in this work.

The paper is organised as follows. In Section. 2, we first introduce the Hawking radiation from a single, isolated black hole. In Section. 3, we discuss generally the exchange of Hawking radiation between two black holes when they get closer and closer. At the final stage of the merger, this leads to non-negligible mass and angular momentum transfer between the holes and causes the black hole parameters to evolve. For a binary system with unequal masses, the evaporation of the light hole will be greatly enhanced in the near-horizon region of the heavy hole. In Section. 4, we argue in the large mass ratio case that the light hole will evaporate completely before reaching the horizon of the latter. In the final section, we summarise and make some extended discussions. 


\section{Hawking radiation of a single black hole}

For a single black hole in empty vacuum, the temperature of Hawking radiation observed at spatial infinity is proportional to the horizon surface gravity: $T_{H}=\kappa / 2 \pi$. The local temperature observed by a stationary observer at any distance larger than the horizon radius is (e.g., [13])

$$
T=\frac{T_{H}}{V}
$$

where the redshift factor $V=\sqrt{\xi_{\mu} \xi^{\mu}}$ with $\xi^{\mu}$ being the Killing vector of the spacetime. $\kappa$ is constant on the orbits of $\xi^{\mu}: \mathcal{L}_{\xi} \kappa=0$. Specifically, it is constant on a stationary horizon, obeying the zero-th law. The Killing vector is null on the horizon. So the local temperature diverges on the horizon.

For a Schwarzschild black hole with mass $\mu$, the surface gravity $\kappa=1 / 4 \mu$ and the redshift factor is

$$
V=\sqrt{1-\frac{2 \mu}{r}} .
$$

The local temperature decreases as the distance increases due to the gravitational redshift $V$. It is approximately equal to $T_{H}$ at a distance of the scale $\sim \mu$ from the horizon. But it becomes large at a distance $r=2 \mu(1+\epsilon)(0 \leq \epsilon \ll 1)$ very close to the horizon:

$$
T \simeq \frac{T_{H}}{\sqrt{\epsilon}}
$$

For a free-fall detector (a probe) whose acceleration near the horizon is

$$
a \rightarrow \frac{\kappa}{V}
$$

it will detect no thermal bath with the temperature (1). In terms of the Unruh effect, it is reasonable to infer that an infalling object moving with a local acceleration less than the one (4) will still see a thermal bath, but with a lower temperature. For example, a massive object that can not be viewed as a probe will not reach the local acceleration (4) near the horizon. So it will see locally a thermal bath with a temperature less than the one given in Eq. (1).

\section{Towards the merger of radiating black holes}

We now discuss generally the merger process of two Hawking radiating black holes with when they move closer and closer. Here, we only consider the non-spinning black holes for simplicity. It is straightforward to extend the results to the spinning case. 
Let us consider the binary black holes with raidii $r_{M}=2 M$ and $r_{m}=2 m$, where $M$ and $m(M \geq m)$ are the masses of the black holes. The black holes still evaporate to future infinity $\mathcal{I}^{+}$. Meanwhile, they can also exchange Hawking radiation between themselves. The radiation from the past horizon $\mathcal{H}^{-}$of one of the holes can go to the future horizon $\mathcal{H}^{+}$of the other hole. During this process, the radiation is not always redshifted, but is first redshifted near the starting hole and then blueshifted near the ending hole. There should be a turning point for this redshift-blueshift transition on each trajectory in between the two holes. The locations of the turning points should change as the binary system evolves. The total redshift effect on a light ray from one horizon to the other is a competing result of the redshift effects around the two holes (notice that the redshift factors may be modified in the binary system) since the redshift factor of one of the holes serves as the blueshift factor for the other hole.

As we shall discuss in detail below, the counter effects can make the Hawking radiation exchanged between black holes more important than the radiation to infinity. For the thermal radiation, we determine the radiation flux by comparing the temperatures, which are more conveniently measured at the turning points (or surface). Since the redshift factors of the two black holes are truncated by each other ${ }^{2}$, the local temperatures observed at a turning point should be larger than the one observed at infinity. They will be large when the separation of two holes becomes very small.

The exchange of Hawking radiation between black holes will induce the evolution of the black hole parameters, like the masses and spins. The thermal particles radiated from one hole can carry some portions of mass and angular momentum away to the other hole. This causes the mass and angular momentum transfer between the black holes, which results in the time-varying parameters according to the first law of mechanics.

\subsection{Large separation analysis}

Initially, the two black holes are separated by a large distance (much greater than their horizon radii), inspiralling around each other with slow velocities. So the black holes may be approximately taken as two isolated holes separated from each other. Their relative motion and tidal interactions can be neglected. In this case, their redshift factors $V_{M}$ and $V_{m}$ simply take the same form (2) as in the isolated case. The radiation following any trajectory from the hole $m$ propagating to the one $M$ is first redshifted and then

\footnotetext{
${ }^{2}$ For a single black hole, the redshift factor varies from 1 to infinity as $r$ decreases from infinity to the horizon radius. In the presence of a companion hole, it can only vary from a value larger than 1 to infinity as $r$ decreases from the turning point to the horizon radius.
} 
blueshifted beyond a turning point.

We consider the turning point on the trajectory that coincides with the straight line crossing both black hole centres, on which the exchange rate of Hawking radiation is the largest. The position of the turning point can be estimated by the balancing condition of the counter redshift effects

$$
V_{M} \simeq V_{m}
$$

Set the separation of the two horizons to be $D$ and the distance of the turning point to the horizon of the hole $m$ to be $d_{T}$. From the condition, the turning position is determined to be at

$$
d_{T} \simeq \frac{m}{M+m} D
$$

So the turning point is closer to the smaller hole. This is also the position where the gravitational forces of the two holes are equal so that a "stationary" observer can reside at this point. As the holes spiral inwards, the turning position changes accordingly. Thus, the observer is not exactly stationary and is taken to be quasi-stationary if the system evolves slowly at large separation. Counting in the rotation of the binary system, this point should be replaced by the first Lagrangian point between the two holes.

At the turning point, the redshift factors are truncated to be

$$
\frac{1}{V_{M}} \simeq \frac{1}{V_{m}} \simeq \sqrt{1+\frac{r_{M}+r_{m}}{D}} .
$$

For finite $D$, the temperatures are larger than the corresponding ones observed at infinity. When $M=m$, the black holes have the same temperature and the net radiation flux between the black holes is zero, i.e., each hole radiates and absorbs the same amount of thermal particles (neglect the radiation to infinity). When the black holes have different masses: $M>m$, the difference of the local temperatures $\Delta T=T^{(m)}-T^{(M)}>0$. This means that there is a net flux of thermal particles from the small hole to the large hole.

As the separation $D$ decreases, the distances $d_{T}$ and $D-d_{T}$ to the horizons of the two black holes both decrease. So the redshift factors $V_{m}$ and $V_{M}$ are both more deeply truncated. The local temperatures and their difference at the turning point increase as $D$ decreases. But, in the large separation limit, the temperatures and their difference are very small for massive black holes.

\subsection{Formation of "common envelopes"}

Due to Hawking radiation, a black hole is expected to evaporate completely in a finite time. The lifetime of a black hole is calculated in $[4,5]$ by summing up the emission rates 
of all species of particles that exist in nature. Thermal particles radiated from the horizon will be possibly reflected back by the curvature of the black hole spacetime. This gives rise to an absorption cross section for each kind of the particles, which characterises a black sphere. The black body radiation of a kind of particle is viewed to be emitted from the surface of the black sphere. At high energies, the radius of the black sphere for all kinds of particles is $\sqrt{27} \mu$ for a hole with mass $\mu$.

In a binary system, either hole contains a "black sphere" whose shape may be modified. When the separation $r_{M}+r_{m}+D$ between the black holes becomes smaller than the total radii of the two black spheres, there should form a "common envelope" filled with the corresponding particles. This common envelope is like the one formed in binary systems of ordinary stars when their Roche lobes are both filled with stellar material. Within the black hole common envelope, the particles radiated from one of the holes are possibly absorbed by itself or by the companion hole. If the black holes have different temperatures, the low-temperature (high-temperature) hole will absorb more (less) particles than it

radiates. But the communication rate of particles in the common envelope is still low if the separation $D$ is not small enough.

\subsection{The merger of the "temperature-divergent" horizons}

With the distance decreasing, the above analysis based on isolated black holes is not applicable. In this case, the dynamics of the system is non-perturbative and is usually studied numerically. The black holes may attain relativistic velocities and the tidal interactions between them become strong. It is found in numerical studies that delicate phenomena emerge in this process $[14,15,16,17,18]$.

In this case, the radiation between the black holes will be complicated, which is not achievable at the moment since the highly dynamical spacetime is unknown. Here, we make only some simple analysis by still approximately taking the black holes as ordinary moving and radiating objects. During the merging process of the horizons, the exchange of Hawking radiation should be very strong because the local temperatures become extremely high approaching the horizons (Note that the horizons are deformed and develop spikes during this process $[14,15,16,17])$. Then the communication rate of Hawking radiation particles between the two holes will become infinitely large, if the semi-classical theory is still trustable in this case. This process proceeds till the merging horizons reach a thermally equilibrium horizon with unique temperature.

Hence, the mass and angular momentum transfer between the holes will be very strong if the divergence of the local temperatures can not be avoided. It was shown that, in the 
presence of a companion object, the black hole will be cooled by the tidal interactions $[19,10]$. In what follows, we shall argue that the tidal interactions and relative motion between black holes are unable to effectively attenuate the extremely high exchanging rate of Hawking radiation.

\subsubsection{Tidal force}

In the binary system, the surface gravity of a black hole is in opposite direction to the tidal force from its companion hole. So the surface gravity or Hawking temperature may be lowered due to the tidal interactions. But, as we argue below, this is not enough to avoid the divergence of the temperatures and their difference on the touching horizons.

First, the divergence of the temperatures on the horizons should not be avoided by tidal interactions. The tidal force by the companion black hole can not reduce the surface gravity on the horizon to zero because zero temperature is not achievable according the third law of thermodynamics. For a non-zero temperature, the local Hawking temperature on the horizon is still divergent since the Killing vector in the redshift factor is null on the horizon.

Second, the huge difference of the local temperatures between merging black holes can not be narrowed down by the tidal forces. The radius of curvature near a black hole with mass $\mu$ is $\mathcal{R} \simeq\left(r / r_{H}\right)^{3 / 2} r_{H}$, where the radius of the horizon $r_{H}=2 \mu$. So, in the binary system, the tidal force on the heavy hole by the light hole is stronger than the reverse one. It has been examined in the numerical simulations for extreme mass ratio systems [15]. The light black hole moving near the large black hole horizon is just like that it moves in a flat spacetime since the curvature near the heavy hole is quite small, while the horizon of the heavy hole is strongly deformed by the light black hole. This means that the heavy hole will be cooled more than the light one. Thus, the tidal interactions will increase the temperature difference rather than smooth it.

\subsubsection{Motion}

The relative motion includes two aspects: the acceleration and velocity.

\section{- Acceleration}

The acceleration of the detector is crucial for it to detect the thermal particles near the horizon. Observers moving with different accelerations will see thermal bath of different temperatures, as stated in the previous section. A stationary observer hovering near horizon will see a thermal bath with the local temperature (1), while a free-fall probelike observer whose acceleration is given by (4) will see nothing at all when crossing the 
horizon. The situation for two black holes is different.

When the two black holes have comparable masses, the smaller hole $m$ can not be viewed as a probe of the larger hole $M$. So its free-fall acceleration can not reach the one (4) locally when its horizon starts to touch the one of the larger hole (Moreover, at the moment, the center of the hole $m$ is $\sim r_{m}$ away from the horizon of the latter, which also greatly suppresses its near-horizon acceleration). For this case, the free-fall small hole $m$ will still "see" a thermal bath, but with a temperature somehow lower than (1). But this can not remove the divergence of the temperature though it is lower.

When the mass ratio is large $M \gg m$, the smaller hole can be viewed as a probe. When it freely collapses towards $M$, it will see no particles when crossing the horizon of $M$. But a stationary observer hovering over the horizon of $M$ (the larger hole is almost static since $M \gg m$ ) can see that the smaller hole $m$ is radiating particles. The acceleration of the observer, together with $M$, moving towards $m$ is equal to the one of $m$ falling towards $M$ and it is much less than the intrinsic near-horizon acceleration (given by Eq. (4)) of a probe falling towards $m$. So the observer can see the hole $m$ is radiating at almost the temperature (1) locally.

\section{- Velocity}

The relative velocity is also important for the observed radiation. The temperature $T=T_{H}$ from Eq. (1) is the one of a single black hole observed by a static observer at spatial infinity. We now suppose that the black hole is moving towards the observer or equivalently the observer is moving towards the black hole with a constant velocity. Then the observed radiation is boosted by a Doppler factor.

This situation also occurs between the inspiral black holes. At their merging stage, the relative velocity between the black holes can reach the relativistic regime. For example, the relative velocity (divided by the speed of light) in the first observed GW event [1] is nearly $\beta \sim 0.6$ at the end of the merger. The relative velocity between the holes will provide an extra redshift factor for the Hawking radiations exchanged between themselves.

We may estimate the effect by recalling the case of the radiation from a radiating and moving source in Minkowski spacetime, though the spacetime in our case is highly curved. The Doppler factor for the observed radiation by a static observer is:

$$
\mathcal{D}=\frac{1}{\Gamma(1-\beta \cos \theta)}
$$

where $\beta$ is the velocity of the radiating source relative to the observer and $\Gamma=1 / \sqrt{1-\beta^{2}}$ is the Lorentz factor. The angle $\theta$ is the one between the moving direction of the source and the line of sight of the observer. The observer will see that the temperature is shifted 
by $T \rightarrow \mathcal{D} T$. In our case, we can choose one of the black hole as the observer and the other as the moving and Hawking radiating source.

For head-on collision of the black holes, the angle is $\theta=0$. The Doppler factor is $\mathcal{D}=(1+\beta) \Gamma$. So the Hawking radiation from one of the holes to the other is boosted in head-on collision.

For the circular rotation case, the angle is $\theta=\pi / 2$ and so the Doppler factor is $\mathcal{D}=1 / \Gamma$. In this case, the Hawking radiation between the holes is de-boosted. But this redshift effect is finite. For binary black holes with relative velocity $\beta=0.9$, the Doppler factor is not quite different from the case with zero velocity. Even for the ultra-high velocity $\beta=0.99$, the Lorentz factor is $\Gamma \simeq 7$. But this case seems not easy to happen because it needs tremendous energy to power a massive black hole to reach such a high velocity. So the circular motion is also unable to effectively attenuate the strong Hawking radiation exchanged between merging black holes.

\section{The extreme mass ratio limit}

As the Hawking radiation is exchanged, there should exist mass and angular momentum transfer between the black holes, which causes the black hole parameters to evolve. To access the details of the evolution of the parameters, numerical calculations are needed for the general case. But the discussion may be simplified in the case for two nonspinning black holes in the extreme mass ratio limit, in which we could take the probe approximation. This case is applicable to the binary systems of a supermassive galactic black hole orbited by a stellar black hole, which are targets of future LISA detectors [20].

For a large mass ratio, the background spacetime is dominated by the one of the heavy hole and is perturbed by the light hole at the order $\mathcal{O}\left(m^{2}\right)$ [21]. The light hole looks like a particle of size $\sim m$ immersed in the background spacetime and it only governs the spacetime that is close to it [15]. In the probe limit, we assume that the spacetime of the heavy hole is rigid and unaffected by the light hole. We ignore the tidal interactions between the holes, which actually increases the difference of the local temperatures as argued in the previous section, and the horizon deformations $[14,15,16,17]$ when the horizons moves towards touching each other.

We shall mainly focus on the process that happens near the horizon of the heavy hole. This region is within the innermost stable circular obit (ISCO), beyond which the light hole will plunge into the heavy hole. Its velocity should be radially dominated. In this case, the relativistic motion can actually boost the exchange rate of Hawking radiation. But, we here ignore this Doppler boosting effect from the relative motion. 
As analysed in Section. (3.3.2), the free-fall hole $m$ will receive almost nothing in the near-horizon regions of the heavy hole $M$. We only need to consider the radiation received by the heavy hole from the light one. With the above approximations, the turning point between the holes (we only consider one turning point, through which the exchange rate is the largest) could be determined by the counter redshift effects from the two Schwarzschild black holes. The redshift factors take the same form as given in Eq. (7). With large mass ratio, the turning position is at

$$
d_{T} \simeq \frac{r_{m}}{r_{M}} D
$$

The local temperature of the light hole with the redshift factor at the turning point is

$$
T^{(m)} \simeq T_{H}^{(m)} \sqrt{1+\frac{r_{M}}{D}}
$$

The temperature $T_{H}^{(m)}$ is associated with the intrinsic surface gravity of the light hole measured in the isolated case.

When $D \gtrsim r_{M}$, the local temperature is nearly equal to $T_{H}^{(m)}$. When $D \ll r_{M}$, the local temperature can be high: $T^{(m)} \simeq \sqrt{r_{M} / D} T_{H}^{(m)}$. For example, the mass ratio of a galactic black hole to a stellar one can reach $M / m=10^{7}$. The local temperature with a separation $D=10 r_{m}$ is $10^{3}$ times larger than $T_{H}^{(m)}$. This means that its evaporation rate is almost $10^{12}$ times larger.

As the light hole moves closer to the heavy hole, the evaporation becomes more strongly enhanced since the redshift factor is more deeply truncated. It will lose mass more quickly, which makes it even hotter. The local temperature (10) diverges at the horizon of the large hole $(D \rightarrow 0)$, consistent with the analysis in the previous section. Thus, the light hole will finally evaporate completely before reaching the horizon of the heavy hole since the evaporation rate diverges there.

This can be clearly seen with some simple calculations. Near the horizon of the heavy hole, the light hole moves at relativistic speed and so $D \simeq-t+$ const with the local time interval $d \tau=V_{M}\left(d_{T}\right) d t$. Then the evolving equation of the mass of the light black hole towards the merger with the heavy hole can be expressed as

$$
\frac{d m}{d D} \propto \delta \Omega r_{m}^{2} T_{H}^{(m)^{4}}\left(\frac{r_{M}}{D}\right)^{\frac{3}{2}}
$$

where $\delta \Omega$ is the solid angle of the Hawking radiation from the the light hole that is effectively enhanced. In the large mass ratio limit, the horizon of the heavy hole looks likes a plane compared with the light hole. So we may assume that the effective solid angle $\delta \Omega$ would be almost the same for the light hole with different mass $m$. 
We can set the mass $m$ at some initial point $D=D_{0}$ as $m_{0}$. The mass becomes $m_{1}$ at point $D=D_{1}\left(D_{0}>D_{1}\right)$. Then we have from the integration of the above equation:

$$
m_{1}=\left[m_{0}^{3}-K\left(\frac{1}{\sqrt{D_{1}}}-\frac{1}{\sqrt{D_{0}}}\right)\right]^{\frac{1}{3}},
$$

where $K$ is a positive constant. So the mass decreases as the separation $D$ decreases. The light hole will evaporate completely with $m_{1}=0$ at

$$
D_{1}=\left(\frac{1}{\sqrt{D_{0}}}+\frac{m_{0}^{3}}{K}\right)^{-2},
$$

before the horizon of the heavy hole is reached (i.e., $D_{1}>0$ ). The evaporated particles are mainly absorbed by the large hole. Note that this locally enhanced evaporation proceeds near the horizon of the heavy hole and it may still remain weak as observed by an observer at infinity due to the huge gravitational redshift. But the observer can sense the occurrence of this mass transfer process by observing the dynamics and GW emission of the binary system.

The result is quite different from that in classical theory, but is similar to the speculations in the Rindler/Tachyon correlation proposed in our previous works [22, 23] (and its extension to the dS space [24]). The mysterious similarity between the action of a probe particle in Rindler space (the geometry near the horizon of a non-extreme black hole) and the tachyon effective action may suggest that the collapsing process of matter towards a black hole horizon is a tachyon condensation process, with the Hawking temperature equal to the Hagedorn temperature in tachyon field theory. The self-energy of the particle will leak to the background spacetime and will be absorbed by the black hole as it moves toward the horizon. In terms of the tachyon field theory, the particle will eventually decay completely into massless and massive closed strings (or gravitons in pure gravity) before reaching the horizon. Our discussion here thus extends this suggestion to the case of particle-like black holes: a light hole moving towards a massive hole will evaporate or decay completely before reaching the horizon. The difference is that here the "particle" is radiating and has temperature.

It is unknown whether the result holds beyond the large mass ratio limit. We guess so if the divergence of the temperature (difference) can not be avoided. But this awaits for critical examinations in full-theory numerical simulations. 


\section{Conclusions and discussions}

We consider the merger process of binary black holes when Hawking radiation is involved. The black holes in a binary system should radiate to each other, leading to mass and angular momentum transfer between them. This quantum effect could be prominent in two situations. One is the case that at least one of the black holes has a high temperature (like the low-mass PBHs [25]). The other is at the final moments of the merging process of general black holes.

As learnt from common sense, the exchange of Hawking radiation particles, which include all kinds of particle in nature, will exert a force or a torque on each of the black hole. This torque tends to slow down the rotation of the binary system so that the black holes can more effectively get closer. The force is the Casimir style since the creation of thermal particles from the event horizons resembles the one on a moving mirror via the dynamical Casimir effect [26] (they may be essentially the same [27]). The CasimirPolder force between black holes due to the exchange of gravitons have been discussed in $[28,29]$. It is also expected that the Casimir force between black holes due to exchange of photons could exist since the black hole horizons can be viewed as conductors with surface resistivity $4 \pi=377 \mathrm{ohm}[30,31,12]$.

The exchange rate of radiation will get extremely large when the temperature-divergent horizons are penetrating each other, even for the binary black holes with the same temperature. We argue that the divergence of the Hawking temperature on the horizons is unable to be attenuated by tidal interactions and relative motion between the black holes. For binary black holes with different temperatures, the difference of the local temperatures will diverge once their horizons touch, which implies that the horizons will instantly evolve into a thermal equilibrium horizon due to the huge difference of the temperatures. The divergence may be hidden from far observers by a common outer horizon formed before the black hole horizons touch each other $[32,18]$ in a highly dynamical system.

We find that it is interesting to discuss the merger of binary black holes with large mass ratio. In the binary system, the evaporation of the light hole is greatly enhanced in the background spacetime of the heavy hole. When the light hole moves in the large spacetime of the heavy hole, it will probably evaporate completely before reaching the horizon of the latter. This is surprisingly consistent with our speculations of the Rindler/Tachyon equivalent descriptions.

The observation of the GWs provides a unique method to probe the nature of black holes and to test gravities beyond GR (e.g., see the reviews [33, 34]). The exchange of Hawking radiation will cause the parameters of the black holes to evolve, which will make 
the GW waveforms different from those predicted in classical theory of gravity ${ }^{3}$. It is hopeful that we may test it in future precise GW observations. However, the effect is prominent only at final stages of the merging process of the horizons, which lasts for a short timescale. Moreover, the inferred masses (and spins) of the black holes from the signal waveforms are not well determined (at a degree of $90 \%$ for the first observed event [2]). All these challenge the test of Hawking radiation in GW observations.

The situation may be improved in binary systems with large mass ratios, which are potential arena for testing fundamental physics $[35,36]$. We have shown that the light hole would significantly lose mass when approaching the near-horizon region of the heavy hole. We also show that the light hole whose evaporation is greatly enhanced will completely evaporate into the heavy hole finally before reaching the horizon of the latter. So it is possible to explore the information loss paradox in the GW observations from such binary systems since the the evolution of the light hole during its whole life is recoded in the GW signals. People have long been puzzled by the information loss problem. When matter collapse into a black hole, its information is lost forever, which violates the laws of quantum mechanics. Many theories have been proposed to solve the problem [37, 38, 39, 40]. Some of them are related to the final stage of the evaporation at the Planck scale, at which a remnant is left or the hole bursts. This could be observable via GWs if the light hole indeed "dissolves" into a large hole.

Before making the precise judgement, we need first to develop detailed theories on the merger process of radiating black holes and produce the corrected waveforms of GWs with numerical relativity in the strong gravity regime.

\section{Acknowledgements}

This work is supported by the Yunnan Natural Science Foundation 2017FB005 and 2014 FB188.

\section{References}

[1] LiGO Scientific, Virgo collaboration, B. P. Abbott et al., Observation of Gravitational Waves from a Binary Black Hole Merger, Phys. Rev. Lett. 116 (2016) 061102, [1602.03837].

\footnotetext{
${ }^{3}$ For example, at the final stage of the merger of two Schwarzschild black holes, the stain of the GW involving Hawking radiation effect should be less than the one predicted in the classical theory since the discrepancy of their masses becomes larger as the radiation exchange proceeds.
} 
[2] Ligo Scientific, Virgo collaboration, B. P. Abbott et al., Properties of the Binary Black Hole Merger GW150914, Phys. Rev. Lett. 116 (2016) 241102, [1602.03840].

[3] S. W. Hawking, Particle Creation by Black Holes, Commun. Math. Phys. 43 (1975) 199-220.

[4] D. N. Page, Particle Emission Rates from a Black Hole: Massless Particles from an Uncharged, Nonrotating Hole, Phys. Rev. D13 (1976) 198-206.

[5] D. N. Page, Particle Emission Rates from a Black Hole. 2. Massless Particles from a Rotating Hole, Phys. Rev. D14 (1976) 3260-3273.

[6] J. L. Friedman, K. Uryu and M. Shibata, Thermodynamics of binary black holes and neutron stars, Phys. Rev. D65 (2002) 064035, [gr-qc/0108070].

[7] K. Uryu, E. Gourgoulhon and C. Markakis, Thermodynamics of magnetized binary compact objects, Phys. Rev. D82 (2010) 104054, [1010.4409].

[8] A. Le Tiec, L. Blanchet and B. F. Whiting, The First Law of Binary Black Hole Mechanics in General Relativity and Post-Newtonian Theory, Phys. Rev. D85 (2012) 064039, [1111.5378].

[9] A. Zimmerman, A. G. M. Lewis and H. P. Pfeiffer, Redshift factor and the first law of binary black hole mechanics in numerical simulations, Phys. Rev. Lett. 117 (2016) 191101, [1606.08056].

[10] A. Le Tiec and P. Grandclement.

[11] S. B. Giddings, Observational strong gravity and quantum black hole structure, Int. J. Mod. Phys. D25 (2016) 1644014, [1605.05341].

[12] K. S. Thorne, R. H. Price and D. A. Macdonald, eds., BLACK HOLES: THE MEMBRANE PARADIGM. 1986.

[13] R. M. Wald, General Relativity. Chicago Univ. Pr., Chicago, USA, 1984, 10.7208/chicago/9780226870373.001.0001.

[14] R. Hamerly and Y. Chen, Event Horizon Deformations in Extreme Mass-Ratio Black Hole Mergers, Phys. Rev. D84 (2011) 124015, [1007.5387]. 
[15] R. Emparan and M. Martinez, Exact Event Horizon of a Black Hole Merger, Class. Quant. Grav. 33 (2016) 155003, [1603.00712].

[16] U. Hussain and I. Booth, Deformation of horizons during a merger, Class. Quant. Grav. 35 (2018) 015013, [1705.01510].

[17] R. Emparan, M. Martinez and M. Zilhao, Black hole fusion in the extreme mass ratio limit, Phys. Rev. D97 (2018) 044004, [1708.08868].

[18] D. Pook-Kolb, O. Birnholtz, B. Krishnan and E. Schnetter, Interior of a Binary Black Hole Merger, Phys. Rev. Lett. 123 (2019) 171102, [1903.05626].

[19] S. E. Gralla and A. Le Tiec, Thermodynamics of a Black Hole with Moon, Phys. Rev. D88 (2013) 044021, [1210.8444].

[20] LISA collaboration, P. Amaro-Seoane et al., Laser Interferometer Space Antenna, 1702.00786 .

[21] S. L. Detweiler, Radiation reaction and the selfforce for a point mass in general relativity, Phys. Rev. Lett. 86 (2001) 1931-1934, [gr-qc/0011039].

[22] H. Li, Strings from geometric tachyon in Rindler space and black hole thermodynamics, JHEP 1203 (2012) 003, [1112.4599].

[23] H. Li, Can matter really cross a horizon?, Int. J. Mod. Phys. D23 (2014) 1442021.

[24] H. Li and J. X. Lu, Tachyon field theory description of (thermo)dynamics in $d S$ space, JHEP 01 (2016) 022, [1511.02078].

[25] D. N. Page and S. W. Hawking, Gamma rays from primordial black holes, Astrophys. J. 206 (1976) 1-7.

[26] B. S. DeWitt, Quantum Field Theory in Curved Space-Time, Phys. Rept. 19 (1975) 295-357.

[27] R. M. Nugaev and V. I. Bashkov, PARTICLE CREATION BY A BLACK HOLE AS A CONSEQUENCE OF THE CASIMIR EFFECT, Phys. Lett. A69 (1979) $385-386$.

[28] L. H. Ford, M. P. Hertzberg and J. Karouby, Quantum Gravitational Force Between Polarizable Objects, Phys. Rev. Lett. 116 (2016) 151301, [1512.07632]. 
[29] J. Hu and H. Yu, Gravitational Casimir-Polder effect, Phys. Lett. B767 (2017) 16-19, [1605.02193].

[30] R. L. Znajek, The electric and magnetic conductivity of a Kerr hole, Mon. Not. Roy. Astron. Soc. 185 (1978) 833-840.

[31] T. Damour, Black Hole Eddy Currents, Phys. Rev. D18 (1978) 3598-3604.

[32] P. Anninos, D. Bernstein, S. R. Brandt, D. Hobill, E. Seidel and L. Smarr, Dynamics of black hole apparent horizons, Phys. Rev. D50 (1994) 3801-3815.

[33] E. Berti, K. Yagi and N. Yunes, Extreme Gravity Tests with Gravitational Waves from Compact Binary Coalescences: (I) Inspiral-Merger, Gen. Rel. Grav. 50 (2018) 46, [1801.03208].

[34] L. Barack et al., Black holes, gravitational waves and fundamental physics: a roadmap, Class. Quant. Grav. 36 (2019) 143001, [1806.05195].

[35] E. Berti et al., Tests of General Relativity and Fundamental Physics with Space-based Gravitational Wave Detectors, 1903.02781.

[36] C. P. L. Berry, S. A. Hughes, C. F. Sopuerta, A. J. K. Chua, A. Heffernan, K. Holley-Bockelmann et al., The unique potential of extreme mass-ratio inspirals for gravitational-wave astronomy, 1903.03686.

[37] J. Preskill, Do black holes destroy information?, in International Symposium on Black holes, Membranes, Wormholes and Superstrings Woodlands, Texas, January 16-18, 1992, pp. 22-39, 1992, hep-th/9209058.

[38] J. Polchinski, The Black Hole Information Problem, in Proceedings, Theoretical Advanced Study Institute in Elementary Particle Physics: New Frontiers in Fields and Strings (TASI 2015): Boulder, CO, USA, June 1-26, 2015, pp. 353-397, 2017, 1609.04036, DOI.

[39] W. G. Unruh and R. M. Wald, Information Loss, Rept. Prog. Phys. 80 (2017) 092002, [1703.02140].

[40] D. Marolf, The Black Hole information problem: past, present, and future, Rept. Prog. Phys. 80 (2017) 092001, [1703.02143]. 